\title{
Heterogeneous trajectories of problematic alcohol use, depressive symptoms, and their co-occurrence in young adults with and without childhood ADHD
}

\author{
Frances L. Wang ${ }^{1}$, Sarah L. Pedersen ${ }^{1}$, Bernie Devlin ${ }^{1}$, Elizabeth M. Gnagy², William E. \\ Pelham Jr. ${ }^{2}$, Brooke S.G. Molina ${ }^{1}$ \\ ${ }^{1}$ University of Pittsburgh, Department of Psychiatry \\ ${ }^{2}$ Florida International University, Department of Psychology
}

\section{Abstract}

The literature is inconsistent regarding whether childhood ADHD confers risk for adulthood problematic alcohol use, depressive symptoms, and their co-occurrence. These inconsistencies could be due to meaningful heterogeneity in the adulthood outcomes of children with ADHD that were obscured in traditional group-based analyses. The current study tested this possibility, as well as the contribution of adulthood ADHD symptom persistence, in order to clarify long-term risk in this population. Children diagnosed with ADHD and demographically-similar children without ADHD were followed longitudinally into adulthood and repeatedly assessed on heavy drinking, alcohol problems, and depressive symptoms from ages 21-29 (84.1\% White (not Hispanic); 86.9\% male; $51.2 \%$ childhood ADHD; $14.7 \%$ adulthood-persistent ADHD; $N=320$ ). Group-based multi-trajectory modeling identified six groups with different combinations of trajectories across these variables. Heterogeneous longitudinal outcomes for those with ADHD were found. Some children with ADHD showed increased risk as typically predicted, with a higher likelihood of membership in a group with stable-moderate alcohol outcomes and stable-severe depression (adulthood persistent ADHD also predicted this group), whereas some children with ADHD were more likely to belong to a group with virtually no alcohol outcomes and low depression. Additionally, adulthood persistent ADHD predicted membership in a group with stable-severe alcohol outcomes and stable-moderate depression. Given the severity associated with co-occurring alcohol and depressive disorders, studies of early risk and protective factors and long-term outcomes for these disparate trajectory patterns are needed, particularly for those with childhood and persisting ADHD.

\footnotetext{
Terms of use and reuse: academic research for non-commercial purposes, see here for full terms. http://www.springer.com/gb/openaccess/authors-rights/aam-terms-v1

Correspondence should be addressed to Frances Wang, Department of Psychiatry, University of Pittsburgh, 3811 O’Hara St., Pittsburgh, PA, 15213. wangfl@upmc.edu.

Author Contributions: Conceptualization: Frances Wang, Brooke Molina, Sarah Pedersen; Formal Analysis: Frances Wang and Bernie Devlin; Data Curation: Elizabeth Gnagy; Funding Acquisition: Brooke Molina, Sarah Pedersen, William Pelham Jr.; WritingOriginal Draft: Frances Wang; Writing: Review \& Editing: Frances Wang, Brooke Molina, Sarah Pedersen, Bernie Devlin, Elizabeth Gnagy.

Conflict of Interest: The authors declare that they have no conflict of interest.
}

Publisher's Disclaimer: This Author Accepted Manuscript is a PDF file of a an unedited peer-reviewed manuscript that has been accepted for publication but has not been copyedited or corrected. The official version of record that is published in the journal is kept up to date and so may therefore differ from this version. 


\section{Keywords}

ADHD; Alcohol; Depression; Comorbidity; Trajectory Groups

Attention-Deficit/Hyperactivity Disorder (ADHD) is a heterogeneous disorder emerging in childhood that includes varying levels of impulsivity, hyperactivity, and inattentiveness (American Psychiatric Association, 2013). ADHD has been conceptualized as an important risk factor for problematic drinking and depression in adulthood, which are severe conditions that together have been shown to result in greater suicide attempts and alcohol severity, and poorer functioning and life satisfaction than either condition alone (Brière et al., 2014). Indeed, children with ADHD are disinhibited by definition, which is a trait with robust risk for alcohol problems (Tarter et al., 2003). Children with ADHD also routinely have poorer academic and relational functioning (Barkley et al., 2006) and emotion regulation (Pedersen et al., 2016) relative to children without ADHD, which may all contribute to greater depressive symptoms (Semeijn et al., 2015).

A relatively large literature has examined whether, as a whole, children with ADHD are at greater risk for future problematic alcohol use or depressive symptoms compared to those without ADHD (e.g., Charach, Yeung, Climans, \& Lillie, 2011; Lee, Humphreys, Flory, Liu, \& Glass, 2011; Meinzer, Pettit, \& Viswesvaran, 2014). However, the results of these studies have been inconsistent. Given the variability within individuals diagnosed with ADHD (Karalunas et al., 2014), the prior inconsistent literature could owe to the presence of unmodeled multifinality in the adulthood outcomes of those with ADHD. Unfortunately, few studies have examined whether those with ADHD show substantively meaningful differences from one another in trajectories of problematic drinking and depression over time. Studies of this nature are needed to clarify whether some individuals with ADHD follow a severe pathway to problematic alcohol use and depressive symptoms and should be targeted for early intervention.

\section{Childhood ADHD is Inconsistently Associated with Adulthood Alcohol Use and Depression}

Studies examining the associations between childhood ADHD and future problematic alcohol use or depressive symptoms have produced mixed outcomes, highlighting the possibility that only certain groups of individuals with ADHD may be at risk (e.g., Charach, Yeung, Climans, \& Lillie, 2011; Lee, Humphreys, Flory, Liu, \& Glass, 2011; Meinzer, Pettit, \& Viswesvaran, 2014). For instance, associations between childhood ADHD and depressive symptoms in both childhood and adulthood have been inconsistent, with some studies showing a positive association (Biederman et al., 2006; Meinzer et al., 2014, 2016) and others showing none (Claude \& Firestone, 1995; Mannuzza, Klein, Bessler, Malloy, \& LaPadula, 1998; Weiss, Hechtman, Milroy, \& Perlman, 1985). A meta-analysis on children with ADHD even suggested a negative association with depression after aggregating only prospective study findings (Meinzer et al., 2014), which highlights the necessity of studying this question longitudinally. With regards to substance use, two meta-analyses have shown that childhood ADHD was overall positively associated with alcohol use disorder in 
adolescence through adulthood (Charach et al., 2011; Lee et al., 2011). However, the effect sizes across studies included in Lee et al. (2011) showed some evidence of heterogeneity, which was not explained by differences across studies such as sex, race, diagnostic instrument, or sample source.

A similar pattern emerges in studies on depression-alcohol co-occurrence. For instance, Biederman et al. (1995) found that substance use disorder and depression were associated cross-sectionally for adults without a history of ADHD, but not for adults with both childhood-onset and past year ADHD. Using a parallel process longitudinal growth model, another study using the Pittsburgh ADHD Longitudinal Sample (PALS) found a positive association between the slopes of heavy drinking and depressive symptoms in the late 20 s that was equally strong for those with or without childhood or adulthood-persistent ADHD (Wang et al., 2019). Thus, the role of childhood ADHD in co-occurring alcohol outcomes and depression remains inconclusive.

\section{Heterogeneity in Adulthood Outcomes among Children with ADHD}

An underexplored possibility is that prior inconsistencies in the literature are due to substantively important differences in the adulthood multivariate, longitudinal outcomes of children with ADHD. Indeed, prior research corroborates the notion that those with childhood ADHD may show meaningful and systematic differences from one another with respect to their adulthood problematic alcohol use and depressive symptoms. In 13-23 year old female twins, Neuman et al. (2001) found evidence for three distinct and heritable subtypes of ADHD that were distinguishable by levels of comorbid disorders, including one showing higher and one showing lower symptoms of major depressive disorder. This suggests that children diagnosed with ADHD are likely to differ in systematic ways from one another with respect to depression, and in ways that would be obscured in traditional between-person analyses. Molina et al. $(2012,2014)$ found similar patterns with regards to alcohol use in the PALS sample such that, relative to a nonADHD group, childhood ADHD indirectly predicted lower levels of heavy drinking at ages 17 and 20 through social impairment but predicted higher levels of heavy drinking at these ages through delinquency. The authors hypothesized that some individuals with ADHD may show lighter drinking because social impairments could preclude involvement in a heavy social drinking network, which is a robust risk factor for problematic drinking during adolescence and young adulthood (Pandina et al., 2010). Using the same sample, Merrill et al. (2019) found evidence for a similar pattern into age 25 . Latent class analyses among only those with childhood ADHD revealed groups that differed on problematic drinking outcomes as well as other functional impairments (depressive symptoms were not examined). Finally, although Wang et al. (2019) found an equally strong positive covariance between alcohol use and depression slopes for children with and without ADHD in the PALS sample, the slopes of depression in adulthood for children with ADHD showed statistically significant variability, as well as greater overall means than for children without ADHD. Thus, findings provided preliminary evidence that there may be a subset of children with ADHD who show stronger co-growth in alcohol use and depression than those without. However, that study was not focused on examining the presence of heterogeneous alcohol-depression trajectories, leaving these conclusions as speculative. 
Thus, there is evidence that those with ADHD show heterogeneity in problematic alcohol use and depressive symptoms in adolescence and adulthood. Surprisingly, to our knowledge, no studies have charted the longitudinal courses of these outcomes throughout the 20s (i.e., 21-29 in this study), examined how these trajectories may co-occur to form unique groups, or related these groups to ADHD diagnoses. Modeling alcohol problems and depression jointly and into the late 20s will add significantly to the current literature on multifinality for children with ADHD. Indeed, the late 20s represents an important developmental period typically marked by influential role transitions, such as marriage and career advancements, which may differently affect trajectories of alcohol use and depression for those with and without ADHD.

This study will also be one of few to shed light on whether children with ADHD are more likely to exhibit alcohol-depression co-occurrence and, if so, the unique patterns of cooccurrence to which they are most susceptible. Indeed, empirical evidence supports the possibility that those with ADHD exhibit unique patterns of co-occurrence. For example, previous work suggested that children with ADHD may be less likely to attain life milestones known to facilitate "maturing out" of alcohol use, such as steady employment and financial independence (Altszuler et al., 2016), as well as to facilitate decreases in depression with age, such as strong social support (Molina et al., 2014). Showing risk for factors that maintain alcohol use and depression over time may suggest that there is a subgroup of children with ADHD that exhibit chronicity in alcohol-depression cooccurrence over the 20s, which is an especially pernicious form of this condition. As another example, a subgroup of children with ADHD could be more likely to show severe depressive symptoms throughout the 20s (e.g., Meinzer et al., 2016), as well as alcohol use that only increases in the late 20s. Indeed, those with ADHD have been shown to exhibit greater levels of negative urgency relative to those without ADHD and could, over time, become more prone to using substances impulsively in response to depressed mood (Pedersen et al., 2016). Based on prior research (Molina et al., 2012), there could also exist an ADHD subgroup showing low levels of alcohol use in the early 20 s, although it is yet unknown the nature of their depressive symptoms.

\section{Importance of Adulthood ADHD Symptom Persistence}

It is also important to consider the role of adulthood ADHD symptom persistence in distinguishing the adulthood outcomes of children with ADHD. ADHD symptoms persist at diagnostic levels into adulthood for only about half of individuals diagnosed with ADHD in childhood (Hechtman et al., 2016). Multiple studies have shown that the persistence of symptoms is associated with worse functioning in adulthood (Barkley et al., 2006;

Hechtman et al., 2016). ADHD symptom persistence may pose a serious risk for problems in adulthood. Continued problems with inhibitory control and attention may have especially debilitating consequences for financial, interpersonal, and psychosocial functioning in adulthood than in earlier stages of life. As impairments in these domains are often stressful, those with adulthood persistent ADHD could show even greater depressive symptoms and heavier drinking to cope when compared to those without (Boden et al., 2014; Kuiper et al., 1986). Moreover, persisting symptoms of impulsivity may continue to impair responsible decision-making with regards to alcohol consumption (Coskunpinar et al., 2013). As such, 
persistence of ADHD symptoms may increase the likelihood of adulthood depression, problematic alcohol use, or both, and could be one factor that explains the heterogeneous outcomes observed among children with ADHD. The second goal of this study was to examine the longitudinal patterns of problematic alcohol use and depressive symptoms from ages 21-29 exhibited by those with adulthood-persistent ADHD (i.e., childhood ADHD alongside 5 or more ADHD symptoms in adulthood).

\section{Current Study}

In the current study, we took advantage of a unique opportunity to parse the heterogeneity in longitudinally tracked depressive symptoms and alcohol problems measured together in young adulthood in PALS - a relatively large longitudinal study that followed children with and without ADHD from childhood to adulthood. In contrast to prior research and other available studies, data were collected over many repeated assessments through this important transition into adulthood when individuals are expected to realize their independence. Thus, the study provides the important opportunity, with conceptually distinct measures of alcohol use not available in most other ADHD studies, to test the co-occurrence of specific trajectories of problem drinking and depressive symptoms and whether they are more, or less, likely as a function of childhood (and persistent) ADHD. Based on prior inconsistencies in the literature, we hypothesized that there would be heterogeneity in the outcomes of those with ADHD. For example, we speculated that one ADHD-associated group could show relatively lower levels of alcohol consumption due to social difficulties that could preclude involvement in a substance-using peer group. However, whether this subgroup would report concurrent depression was unknown. We also hypothesized that one group could show severe and either increasing or chronic levels of all outcomes through the 20s and that this group would also be overrepresented by childhood ADHD, as those with ADHD are at risk for outcomes that may result in the maintenance of alcohol problems and depressive symptoms over time. To uncover these patterns, we used group-based multitrajectory modeling (Nagin et al., 2018), which is an approach that allows the identification of groups differing with respect to their trajectories on multiple outcomes.

\section{Method}

\section{Participants}

Participants were drawn from the Pittsburgh ADHD Longitudinal Study (PALS; 84.1\% White (not Hispanic); $86.9 \%$ male; $N=320$ for trajectory models; $N=316$ for regression analyses due to missingness in one covariate).

Participants with childhood ADHD, n=364, were recruited for longitudinal follow-up from an eight-week ADHD summer treatment program conducted between 1987 and 1996 (Pelham et al., 1996; $M_{\text {age }}=9.4$ years, $S D=2.27$ years, range $=5.0-16.92$ ). Although female children participated in the program, male children predominated. This reflects the significantly higher rates of ADHD found among boys in the population (more than twice as many boys as girls; Cohen et al., 1993). ADHD was diagnosed using DSM-III-R or DSM-IV criteria. Clinicians (Ph.D.-level) interviewed parents using standardized semi-structured diagnostic instruments that included DSM-III-R or DSM-IV criteria for ADHD, oppositional 
defiant disorder, conduct disorder, other Axis I disorders and supplementary questions (available through co-author, W.E.P). Parents and teachers also completed standardized DSM-III-R and DSM-IV symptom rating scales including the Disruptive Behavior Disorders (DBD) Scale (Pelham, Gnagy, Greenslade, \& Milich, 1992). ADHD and conduct disorder for the current study were based on passing DSM thresholds from these assessments. Exclusion criteria were a full-scale IQ $<80$, a history of schizophrenia, pervasive developmental disorder, other psychotic or organic mental disorders, seizures or other neurological problems.

The first follow-up for the participants with childhood ADHD occurred an average of 8.26 years $(S D=2.72)$ after initial assessment. Rolling enrollment occurred between 1999 and 2003. Of those eligible for the initial follow-up $(n=516), 70.5 \%$ participated $(n=364)$.

Those with ADHD who participated had lower conduct disorder symptom ratings than those with ADHD who did not participate, but the mean difference was small $(M=.43$ and .53 , respectively; Cohen's $\mathrm{d}=.30$ ). No differences between groups were found for other demographic, diagnostic, or symptomatology variables.

Participants without ADHD ( $n=240)$, demographically similar to the ADHD group on age, sex, ethnicity and/or race, and parental education, were recruited on a rolling basis during the same time ADHD group participants were recruited into the follow-up study (see Molina et al., 2012 for details). Childhood ADHD and CD were assessed via retrospective reports from probands and parents on the DSM-IV rating scale. Exclusionary criteria listed previously also applied to nonADHD group participants.

At their initial follow up (ADHD group), or during their first assessment (nonADHD group), participants ranged in age from 11-25 years old. Annual assessments were then administered for 9 years. Thereafter, participants were administered age-based assessments corresponding to the ages of $23,25,27,29,35$, and 40 . For the most part, the total number of eligible youth was the same at each age point. However, a small number of participants were not eligible at ages 21 and 23 because they were older than this at their first assessment due to the 11-25 year old age range at first interview.

Current subsample.-Out of 604 participants (364 ADHD, 240 nonADHD), 320 ( $n=164$ ADHD, $n=51.3$ nonADHD; $n=269$ White (not Hispanic); $n=42$ female) were included in the current study because they provided at least 11 out of 15 possible assessments on the three outcome variables at ages $21,23,25,27$, or 29 (i.e., they were missing fewer than 4 , or $25 \%$ of, assessments). This strategy maximized the sample size, while also ensuring that we had enough longitudinal data to estimate trajectory groups. In this sample of 320 participants, the number of assessments completed was not correlated with childhood or adulthood-persistent ADHD. This helps reduce the concern that unequal missingness by ADHD group could bias the estimated trajectory groups and their associations with ADHD. 67 participants provided all 15 possible assessments, 43 provided 14 assessments, 66 provided 13 assessments, 68 provided 12 assessments, and 76 provided 11 assessments. Participants who were included versus excluded in the current study did not differ in sex $\left(\chi^{2}=2.85, p=0.09\right)$ or race $\left(\chi^{2}=\right.$ $1.66, p=0.20)$, but they were less likely to have childhood $\left(\chi^{2}=20.85, p<0.001\right)$ and adulthood-persistent $\left(\chi^{2}=4.59, p=0.03\right)$ ADHD. 
Ethical considerations and procedure.-Participants and parents were interviewed by research staff and completed questionnaires privately. Informed consent was obtained from all individual participants included in the study. Confidentiality was assured except in cases of imminent harm/danger to self or others. Mail and telephone were used when distance prevented office visits. The University of Pittsburgh Institutional Review Board approved this study.

\section{Measures}

Heavy alcohol use.-Participants reported their frequency of past-year heavy drinking ("In the past 12 months, how often did you drink 5 or more drinks when you were drinking?"; 11 options: $0=$ not at all; $1=1-3$ times; $2=4-7$ times; $3=8-11$ times; $4=$ Once a month; $5=2-3$ times/month; $6=$ Once a week; $7=2-3$ times a week; $8=4-6$ times a week; $9=$ once a day; $10=$ Twice a day; $11=$ several times a day). This item was from the Substance Use Questionnaire (Molina \& Pelham, 2003), adapted from the Health Interview Questionnaire(Jessor et al., 1981) and the National Household Survey of Drug Abuse interview. From ages 21-29, heavy alcohol use showed the following ranges: $M=2.37-$ $3.25, S D=2.53-2.87$, Range $=0-11$.

Alcohol problems.-Participants reported their alcohol problems using a 36-item modified version of the Young Adult Alcohol Problems Screening Test (Hurlbut \& Sher, $1992 ; 0=$ none or not in the past year to $3=$ more than 5 times). We coded each item dichotomously to capture number of different alcohol problems in the past year. Responses were summed, as no item-level data were missing. From ages 21-29, number of different alcohol problems showed the following ranges: $M=2.46-4.50, S D=4.22-5.50$, Range $=0$ 36.

Depressive symptoms.-Participants reported their depressive symptoms in the past week using the Center for Epidemiologic Studies Depression Scale (Radloff, 1977; $0=$ rarely or none of the time to $3=$ most or all of the time). Total scores were calculated as the sum of item responses because no item-level data were missing. From ages 21-29, this scale showed the following ranges: $M=11.34-12.15, S D=7.44-9.60$, Cronbach's Alpha $=0.78$ 0.90 .

Adulthood-persistent ADHD.-Participants and their parents reported 18 past-year ADHD symptoms corresponding to $D S M-I V-T R$ symptoms $(0=$ never to $3=$ very often) during selected follow-up visits using the Adult ADHD Rating Scale (Barkley, 2011; Barkley, Murphy, \& Fischer, 2010; Kessler et al., 2010). Adulthood-persistent ADHD was coded "1" if either participants or parents endorsed five or greater ADHD symptoms (consistent with DSM-5) at age 25, 27, or 29 and if they had childhood ADHD (17.6\% of participants met criteria for adulthood-persistent ADHD). All others, including those symptom-desistant by adulthood, were coded " 0. ."

Demographics.-Participants reported their sex and responded to a question about their race and/or ethnicity (84.1\% White (not Hispanic), 10.6\% Black or African American, 1.3\% Hispanic or Spanish American [Mexican, Puerto Rican, Cuban, Latin American], 0.3\% 
Asian American, 3.8\% Mixed race). Those who were White (non-Hispanic) were coded "1" and all other racial and ethnic groups " 0 ." Parents reported their highest level of education at first follow-up $(1=$ partial high school, $2=$ high school graduate or GED, $3=$ technical school, $4=$ college (at least one year), $5=$ two-year college degree, $6=$ four-year college degree, 7 = graduate degree).

\section{Analytic Plan}

Group-based multi-trajectory modeling was conducted using SAS Software, Version 9.4 using PROC TRAJ in the full sample (Nagin et al., 2018). This method identifies latent clusters of individuals following similar trajectories across multiple outcomes and handles missing data. Heavy drinking and depressive symptoms were determined to have censored normal distributions. Alcohol problems were determined to have a zero-inflated Poisson distribution, as the probability of excess zeroes ranged from $0.23-0.40$ (estimated using pscl package in R; Zeilas, Kleiber, \& Jackman, 2008). Graphical displays suggested that the probability of extra-Poisson zeroes should be modeled with a linear function common to all groups.

To approximate the number of groups to test in multi-trajectory models, we determined the best-fitting group-based trajectory model for each outcome separately using the Bayesian Information Criteria (BIC). The four-group models maximized BIC for depressive symptoms and heavy drinking, whereas a six-group model maximized BIC for alcohol problems. We then tested multi-trajectory models with one to eight independent groups. For each model, groups were specified as following quadratic trajectories, but polynomials were trimmed if not statistically significant (to linear or zero-growth functions). Although BIC is a useful tool for screening mixture models, by itself it cannot guarantee selection of the generative model (Nagin et al., 2018). In addition to using BIC, we also examined whether each new group added distinct and meaningful information about the sample population. The final, chosen model was evaluated on indicators of model adequacy, such as meeting established cut-offs for the average posterior probability (>.70) and odds of correct assignment (>5; Nagin et al., 2018).

From the final model we computed the posterior probability of group membership, which quantifies the probability of an individual's membership in each group. Each posterior probability was log-transformed and analyzed separately as a continuous outcome variable of childhood, and separately, adulthood-persistent, ADHD. Covariates included participants' sex, race, childhood conduct disorder (CD), and parental education. Notably, the inclusion of childhood CD as a covariate increases confidence that ADHD, and not this highly comorbid condition, drives risk for alcohol and depression outcomes. Regression analyses were conducted in Mplus v.8.1 (Muthén \& Muthén, 1998-2017) using maximum likelihood estimation. Due to some missing data on parental education $(n=4)$, the sample size for regression analyses was 316 . 


\section{Results}

\section{Zero-order correlations}

(See Table 1). Childhood ADHD was correlated with childhood CD, lower levels of heavy drinking throughout the 20s, fewer alcohol problems from ages 21 to 25, and higher levels of depression at ages 21,23, and 29. Adulthood-persistent ADHD was not correlated with heavy drinking at any age, was correlated with greater alcohol problems at age 29 , and with greater depressive symptoms at all ages but age 27. Autocorrelations ranged from $0.27-0.57$ for depressive symptoms, $0.52-0.75$ for heavy drinking, and $0.34-0.55$ for alcohol problems (all $p$ 's<0.05).

\section{Multi-trajectory models.}

BIC improved with the addition of groups (see Table 2), with substantial changes in fit (i.e., changes in BIC greater than 50; Kass \& Raftery, 1995) occurring up to six groups. Models with more than six groups began to taper off in terms of BIC improvements and the added groups were not substantively meaningful above those in the six-group model. Thus, the sixgroup model was selected. This model met established cut-offs of model adequacy, with the average posterior probability equal to 0.92 and odds of correct assignment ranging from 23.8-466.6. The six-group model contained groups showing: (1) virtually no problems: virtually no heavy drinking or alcohol problems, low depression ( $n=67)$; (2) low problems: low alcohol outcomes and depression, although higher than the former group ( $n=82)$; (3) moderate-decreasing drinkers with lowest depression: moderately-severe and decreasing alcohol outcomes, lowest of all groups in depression ( $n=53)$; (4) severe-decreasing drinkers with low depression: severe and decreasing alcohol outcomes, low depression ( $n=71)$; (5) comorbid primarily depression: moderately-severe and chronic alcohol outcomes, severe and chronic depression ( $n=18$ ); (6) comorbid primarily alcohol: severe, chronic alcohol outcomes, moderately-severe and chronic depression ( $n=29$; Figure 1).

\section{Group membership predictors.}

See Tables 3 and 4 for full results and the effects of covariates on group membership. Those with a higher likelihood of membership in the virtually no problems group were significantly more likely to have childhood ADHD (67.2\%) but less likely to have adulthood-persistent ADHD (16.4\%). Those with a higher likelihood of membership in the low problems group were no more likely to have childhood ADHD (51.2\%) but were significantly less likely to have adulthood-persistent ADHD (11.0\%). Those with a higher likelihood of membership in the moderate-decreasing drinkers with lowest depression group were also no more likely to have childhood ADHD (37.7\%) but were significantly less likely to have adulthood-persistent ADHD (11.3\%). Those with a higher likelihood of membership in the severe-decreasing drinkers with low depression group were marginally significantly less likely to have childhood ADHD (42.3\%) but not less likely to have adulthood-persistent ADHD (8.5\%). Those with a greater likelihood of membership in the comorbid primarily depression group were significantly more likely to have childhood (77.8\%) and adulthoodpersistent ADHD (33.3\%). Those with a greater likelihood of membership in the comorbid primarily alcohol group were no more likely to have childhood ADHD (44.8\%) but significantly more likely to have adulthood-persistent ADHD (31.0\%). 


\section{Post-hoc analyses.}

Among those with childhood ADHD, greater severities of childhood inattentive, hyperactive/impulsive, or ODD/CD symptoms may drive a higher likelihood of membership in the higher risk groups (i.e., comorbid) or a lower likelihood of membership in the lower risk group (i.e., virtually no problems). We tested this possibility by regressing the logtransformed posterior probability of membership for each group on inattentive, hyperactive/ impulsive, and ODD/CD symptoms plus covariates. All three continuous variables were drawn from the DBD scale and calculated as a mean of symptom ratings, with each symptom defined as the highest of parent or teacher ratings (see Molina \& Pelham, 2003). Neither inattentive $(\beta=-0.112-0.112, p=0.169-0.744)$, hyperactive/impulsive $(\beta=$ $-0.050-0.125, p=0.177-0.742)$ nor ODD/CD $(\beta=-0.150-0.025, p=0.097-0.834)$ symptom severity predicted membership in any of the groups.

We also evaluated whether our inclusion criteria or missing data patterns influenced the reliability of trajectory groups. To assess whether excluding participants with more missing data (who were more likely to have ADHD) biased trajectory group results, we estimated a six-group model in which participants who completed at least one assessment were included $(n=538 ; 57.4 \%$ ADHD). These results were virtually identical to the original model (see Supplementary Figure 1). We also conducted analyses to ensure that the trajectory shapes within each group (e.g., stable, decreasing) did not change with differing amounts of missing data. There was no association between trajectory group membership and the number of assessments completed $\left(\chi^{2}=25.97, p=0.17\right)$.

\section{Discussion}

Clarifying whether children with ADHD follow distinct trajectories of problematic alcohol use and depressive symptoms in adulthood could improve accuracy of prognosis and treatment for this prevalent diagnostic population. The current study directly identified distinctive trajectory groups of alcohol problems and depression over time and tested their associations with ADHD. Critically, results from this study suggested that there may be two distinct forms of alcohol-depression comorbidity related to ADHD, which is a completely novel finding that offers new and important insights into adulthood risk for children diagnosed with ADHD. Also novel were results showing that there existed a group with virtually no depressive symptoms or alcohol use that was related to ADHD, as well as developmentally non-normative chronicity in alcohol use and depression in the 20 s for those with ADHD.

Specifically, we found three potentially clinically meaningful groups overrepresented by childhood or adulthood persistent ADHD. Two showed longitudinally co-occurring and chronic alcohol problems and depression and were each distinguished by the primacy of either alcohol problems or depression (comorbid primarily alcohol and comorbid primarily depression), whereas the third showed essentially no problematic alcohol use and low depressive symptoms (virtually no problems). We found additional groups exhibiting initially moderate or high and decreasing alcohol outcomes and low-stable depression (groups 3 and 4), as well as low levels of all problems (group 2), that were overrepresented by participants without ADHD histories. 


\section{Co-occurring Trajectories of Alcohol Problems and Depression for those with ADHD}

Most studies of psychiatric comorbidity with alcohol problems or depression in ADHD have relied on cross-sectional samples and diagnostic histories (current or lifetime) and have not been positioned to study the heterogeneous pairings of these problems over time. By addressing these limitations, our findings strongly suggested that some children with ADHD (based on the current findings $~ 15 \%$ ) have increased risk for co-occurring alcohol problems and depressive symptoms in young adulthood. In fact, with the exception of the virtually no problems group, childhood or adulthood persistent ADHD were only associated with groups characterized by elevations in both types of adulthood problems (i.e., groups 5 and 6), and never showed depressive symptoms or alcohol problems alone. As co-occurring alcohol problems and depressive symptoms have been shown to be associated with functional impairments and poor treatment outcomes (Brière et al., 2014; Modesto-lowe \& Kranzler, 1999), it may be fruitful to target some children with ADHD for both early and continued intervention into adulthood to prevent the co-occurrence of these conditions.

The comorbid primarily depression group (group 5), predicted by childhood ADHD approximately 20 years earlier, was characterized by clinically-meaningful levels of depression (average CES-D scores of 27 at all ages, which exceeds the clinical cut-off of 16; Radloff, 1977), moderately-severe levels of alcohol problems, and light-to-moderate heavy drinking. Persistence of ADHD into adulthood also predicted membership in this group. Interestingly, adults in this group showed a substantial number of alcohol problems in the past year (5-6 problems, on average) despite relatively infrequent heavy drinking (5+ drinks about 4-7 times per year, on average). This subgroup may have had fewer social opportunities for drinking as a consequence of depression. However, when drinking episodes did occur they may have been quite heavy and problematic, perhaps to cope with high levels of negative affect (Cooper et al., 1995).

Children whose ADHD symptoms persisted into adulthood also had a higher likelihood of membership in Group 6 (comorbid primarily alcohol), which was characterized by the most severe levels of problematic alcohol use relative to all other groups, as well as levels of depression that were just short of the clinical threshold (average CES-D scores of 14). This group is unique from the comorbid primarily depression group (group 5) in their primacy of alcohol problems, a pattern potentially suggesting that alcohol problems may drive depression for this subgroup perhaps by increasing life stress (Hart \& Fazaa, 2004). Importantly, the effects of childhood or adulthood persistent ADHD on problematic alcohol use was only revealed in our study by jointly examining depression (e.g., groups 5 and 6). Note that adulthood-persistent ADHD was underrepresented in groups exhibiting moderate and low alcohol problems without accompanying depression (groups 2 and 3) and that childhood ADHD was marginally significantly underrepresented in the group showing high alcohol problems without accompanying depression (group 4). Results thus help resolve prior inconsistencies regarding the associations of childhood ADHD with substance use and depression and suggest that joint consideration of these co-occurring vulnerabilities may be necessary to attain clarity in the literature. 


\section{Role of Adulthood Persistent ADHD Symptoms in Co-occurrence}

Adulthood persistent ADHD was a feature of both comorbid groups. However, note that these associations were not likely due to those with symptom persistence showing more severe symptomatology in childhood, as adulthood persistent ADHD was not correlated with the severity of childhood inattentiveness $(r=-0.02, p=0.77)$, hyperactivity/impulsivity $(r=$ $-0.08, p=0.29)$, or ODD/CD symptoms $(r=-0.02, p=0.77)$. This suggests that continuing symptoms of impulsivity, attentional problems, and/or hyperactivity play a particularly important role in the development of co-occurring alcohol problems and depression (Snyder, 2013; Weafer, Milich, \& Fillmore, 2011). Negative urgency is one facet of impulsivity that is heightened in those with ADHD (Pedersen et al., 2016) and may be particularly salient in explaining the role of ADHD persistence in alcohol-depression co-occurrence. Indeed, individuals high in negative urgency are at greater risk for impulsive, problematic drinking in response to negative affect (Coskunpinar et al., 2013). Although less frequently studied, research also showed that those high in negative urgency were at greater risk for depression because, in the face of negative emotions, these individuals may "rashly" avoid without appropriate consideration of the consequences (Smith, Guller, \& Zapolski, 2013).

Adulthood persistent ADHD may also portend more debilitating functional consequences in a variety of domains relative to ADHD symptoms in childhood or adolescence, as the responsibilities and expectations of adulthood are greater. The stress associated with these consequences may lead to depression or heavy drinking to cope with stress (Boden et al., 2014; Kuiper et al., 1986), potentially explaining why adulthood persistent ADHD played an important role in co-occurring alcohol-depressive symptoms. The contextual influences that lead to adulthood persistent ADHD, such as poor parental mental health (Roy et al., 2016), could also contribute to co-occurrence of alcohol use and depression in adulthood. Results suggest that enhancing the identification of children with ADHD who are most at risk for persisting ADHD symptoms into adulthood could help improve early identification for prevention. Moreover, continued treatment of ADHD symptoms into adulthood may be important.

\section{Childhood ADHD Also Predicts Low Levels of Alcohol use and Depressive Symptoms}

Childhood ADHD also predicted membership in a group exhibiting very low problematic alcohol use and depressive symptoms throughout the 20s (virtually no problems group; reporting, on average, zero heavy drinking episodes and alcohol problems per year, and scoring about 10 on the CES-D, well below the clinical cut-off). The relatively larger size of this ADHD-associated group relative to the comorbid groups explains why there is an overall negative correlation between ADHD and heavy drinking in our sample (see Table 1). Alcohol use in this group was atypically low compared to other samples in this age range (Patrick \& Schulenberg, 2011). Thus, the virtually no problems group could represent a different type of impaired group who may be less socially connected with peers, precluding socially-oriented drinking. This hypothesis is supported by prior research that showed that children with ADHD were more educationally, vocationally and socially impaired and more likely to live at home as adults than those without ADHD (e.g., Barkley et al., 2006; Merrill et al., 2019). Although such impairments may well explain the low levels of alcohol use in this group, they do not provide insight into the low levels of depressive symptoms they 
reported. More research is needed to understand whether individuals in this group possess certain factors that protect them from developing depression.

Collectively, the virtually no problems group (group 1) and the comorbid groups (5 and 6) demonstrate that there are substantively meaningful differences among those with childhood ADHD that may explain previous inconsistent effects of childhood ADHD on alcohol outcomes and depression. An important topic for future research will be to understand the childhood factors that differentiate each of these groups, as this information could identify those in need of early prevention as well as the type of early intervention best suited to each. Notably, our post-hoc work did not identify the severity of childhood ADHD or ODD/CD symptoms as important in differentiating who, among those with ADHD, develop alcoholdepression co-occurrence.

\section{ADHD Predicts Developmentally Non-Normative Trends in Alcohol Use and Depression}

Epidemiological research shows that adults consume less alcohol and experience fewer alcohol-related consequences as they mature toward the end of their 20s (Dawson et al., 2006). Decreases have been attributed to the responsibilities of adulthood, such as marriage and employment, which are at odds with heavy drinking with advancing age (Dawson et al., 2006). Similarly, depressive symptoms decrease throughout adulthood due to positive adaptations of this period, like increased social support and marriage (Galambos et al., 2006).

Our study importantly highlights the developmentally non-normative trends in depression and problematic alcohol use followed in the 20s for those with childhood or adulthood persistent ADHD, including chronically high levels of both problems or unusually low levels of alcohol use. In contrast, groups more heavily comprised of participants without ADHD followed more typical, decreasing trends in alcohol use and relatively stable and low depression levels in the 20s or showed low levels of drinking and depression. Perhaps those with ADHD did not show the typically-observed declines in these conditions because they are less likely than their peers to attain important developmental milestones, like steady employment and financial independence, as we have shown for this sample (Altszuler et al., 2016). From a clinical perspective, our results suggest that fostering the attainment and maintenance of important life milestones could be important treatment goals for those with histories or current symptoms of ADHD. Health care providers should also be aware that, for patients with past and present ADHD symptoms, heavy drinking and depressive symptoms in the early 20s may in fact signal chronicity in these outcomes into the late 20 s rather than developmentally-limited phenomena.

Despite many strengths including the use of prospective young adulthood data, rigorously measured ADHD symptoms in childhood and adulthood, and modeling adulthood outcome heterogeneity, our results should be considered in light of several limitations. Although in this study we focused on alcohol-depression comorbidity due to its high prevalence and particularly poor outcomes, future studies should include other related conditions, such as antisocial behavior and anxiety. We found that female and non-White participants were more likely to belong to groups with lower levels of alcohol problems and drinking. We were under-powered to test whether these characteristics moderate the association between 
ADHD and trajectory group membership; this possibility should be tested in future work. However, our replication of these demographic predictions from research in ADHD populations (e.g., Howard et al., 2015) and extension to older ages is important given the rarity of females and racial and ethnic minorities in longitudinal ADHD samples.

In conclusion, this study contributed importantly to our knowledge of the long-term prognosis for children with ADHD with regard to expensive, impairing mental health outcomes: depression, heavy drinking and alcohol problems. We showed that children with ADHD, especially when coupled with ADHD symptom persistence in adulthood, displayed heightened risk for two potentially distinct forms of alcohol-depression co-occurrence. We also demonstrated that the inconsistent links from ADHD to future alcohol outcomes and depressive symptoms in prior studies may owe, in part, to systematic and substantively important differences in longitudinal pathways among those with childhood ADHD. Uncovering additional early, and intervening, risk factors that distinguish among these subgroups will be crucial to identify those most in need of prevention for severe forms of cooccurrence. Results also highlight the developmentally non-normative pathways that children with ADHD may traverse as adults, warranting additional research to discover the factors that will improve mental health outcomes of these high-risk youths in the future.

Ethical approval: All procedures performed in studies involving human participants were in accordance with the ethical standards of the institutional research committee (University of Pittsburgh Institutional Review Board, \#970723) and with the 1964 Helsinki declaration and its later amendments or comparable ethical standards.

\section{Supplementary Material}

Refer to Web version on PubMed Central for supplementary material.

\section{ack}

Funding: This research was principally supported by AA011873 (Molina) and DA12414 (Pelham). Support was also provided by AA00202 (Molina), AA007453 (Molina, Richardson).

\section{References}

Altszuler AR, Page TF, Gnagy EM, Coxe S, Arrieta A, Molina BS, \& Pelham WE (2016). Financial dependence of young adults with childhood ADHD. Journal of Abnormal Child Psychology, 44(6), 1217-1229. [PubMed: 26542688]

Barkley RA (2011). Barkley Adult ADHD Rating Scale-IV (BAARS-IV). Guilford Press.

Barkley RA, Fischer M, Smallish L, \& Fletcher K. (2006). Young adult outcome of hyperactive children: Adaptive functioning in major life activities. Journal of the American Academy of Child \& Adolescent Psychiatry, 45(2), 192-202. [PubMed: 16429090]

Barkley RA, Murphy KR, \& Fischer M. (2010). ADHD in adults: What the science says. Guilford Press.

Biederman J, Monuteaux MC, Mick E, Spencer T, Wilens TE, Silva JM, Snyder LE, \& Faraone SV (2006). Young adult outcome of attention deficit hyperactivity disorder: A controlled 10-year follow-up study. Psychological Medicine, 36(2), 167-179. [PubMed: 16420713]

Biederman J, Wilens T, Mick E, Milberger S, Spencer TJ, \& Faraone SV (1995). Psychoactive substance use disorders in adults with attention deficit hyperactivity disorder (ADHD): Effects of 
ADHD and psychiatric comorbidity. American Journal of Psychiatry, 152(11), 1652-1658. [PubMed: 7485630]

Boden JM, Fergusson DM, \& Horwood LJ (2014). Associations between exposure to stressful life events and alcohol use disorder in a longitudinal birth cohort studied to age 30. Drug and Alcohol Dependence, 142, 154-160. [PubMed: 25001278]

Brière FN, Rohde P, Seeley JR, Klein D, \& Lewinsohn PM (2014). Comorbidity between major depression and alcohol use disorder from adolescence to adulthood. Comprehensive Psychiatry, 55(3), 526-533. [PubMed: 24246605]

Charach A, Yeung E, Climans T, \& Lillie E. (2011). Childhood attention-deficit/hyperactivity disorder and future substance use disorders: Comparative meta-analyses. Journal of the American Academy of Child \& Adolescent Psychiatry, 50(1), 9-21. [PubMed: 21156266]

Claude D, \& Firestone P. (1995). The development of ADHD boys: A 12-year follow-up. Canadian Journal of Behavioural Science, 27(2), 226-249.

Cohen P, Cohen J, Kasen S, Velez CN, Hartmark C, Johnson J, Rojas M, Brook J, \& Streuning EL (1993). An epidemiological study of disorders in late childhood and adolescence-I. Age-and gender-specific prevalence. Journal of Child Psychology and Psychiatry, 34(6), 851-867. [PubMed: 8408371]

Cooper ML, Frone MR, Russell M, \& Mudar P. (1995). Drinking to regulate positive and negative emotions: A motivational model of alcohol use. Journal of Personality and Social Psychology, 69(5), 990. [PubMed: 7473043]

Coskunpinar A, Dir AL, \& Cyders MA (2013). Multidimensionality in impulsivity and alcohol use: A meta-analysis using the UPPS model of impulsivity. Alcoholism: Clinical and Experimental Research, 37(9), 1441-1450.

Cyders MA, \& Smith GT (2008). Emotion-based dispositions to rash action: Positive and negative urgency. Psychological Bulletin, 134(6), 807. [PubMed: 18954158]

Dawson DA, Grant BF, Stinson FS, \& Chou PS (2006). Maturing out of alcohol dependence: The impact of transitional life events. Journal of Studies on Alcohol, 67(2), 195-203. [PubMed: 16568565]

Galambos NL, Barker ET, \& Krahn HJ (2006). Depression, self-esteem, and anger in emerging adulthood: Seven-year trajectories. Developmental Psychology, 42(2), 350. [PubMed: 16569173]

Hart KE, \& Fazaa N. (2004). Life stress events and alcohol misuse: Distinguishing contributing stress events from consequential stress events. Substance Use \& Misuse, 39(9), 1319-1339. [PubMed: 15462232]

Hechtman L, Swanson JM, Sibley MH, Stehli A, Owens EB, Mitchell JT, Arnold LE, Molina BS, Hinshaw SP, \& Jensen PS (2016). Functional adult outcomes 16 years after childhood diagnosis of attention-deficit/hyperactivity disorder: MTA results. Journal of the American Academy of Child \& Adolescent Psychiatry, 55(11), 945-952. [PubMed: 27806862]

Howard AL, Molina BS, Swanson JM, Hinshaw SP, Belendiuk KA, Harty SC, Arnold LE, Abikoff HB, Hechtman L, \& Stehli A. (2015). Developmental progression to early adult binge drinking and marijuana use from worsening versus stable trajectories of adolescent attention deficit/ hyperactivity disorder and delinquency. Addiction, 110(5), 784-795. [PubMed: 25664657]

Hurlbut SC, \& Sher KJ (1992). Assessing alcohol problems in college students. Journal of American College Health, 41(2), 49-58. [PubMed: 1460173]

Jessor R, Jessor SL, \& Donovan JE (1981). Young adult follow-up study: 1981 annual questionnaire. Institute of Behavioral Science.

Karalunas SL, Fair D, Musser ED, Aykes K, Iyer SP, \& Nigg JT (2014). Subtyping attention-deficit/ hyperactivity disorder using temperament dimensions: Toward biologically based nosologic criteria. JAMA Psychiatry, 71(9), 1015-1024. [PubMed: 25006969]

Kessler RC, Green JG, Adler LA, Barkley RA, Chatterji S, Faraone SV, Finkelman M, Greenhill LL, Gruber MJ, \& Jewell M. (2010). Structure and diagnosis of adult attention-deficit/hyperactivity disorder: Analysis of expanded symptom criteria from the Adult ADHD Clinical Diagnostic Scale. Archives of General Psychiatry, 67(11), 1168-1178. [PubMed: 21041618] 
Kuiper NA, Olinger LJ, \& Lyons LM (1986). Global perceived stress level as a moderator of the relationship between negative life events and depression. Journal of Human Stress, 12(4), 149153. [PubMed: 3559198]

Lee SS, Humphreys KL, Flory K, Liu R, \& Glass K. (2011). Prospective association of childhood attention-deficit/hyperactivity disorder (ADHD) and substance use and abuse/dependence: A metaanalytic review. Clinical Psychology Review, 31(3), 328-341. [PubMed: 21382538]

Littlefield AK, \& Winograd RP (2013). Maturing out In Principles of Addiction (pp. 363-370). Elsevier.

Mannuzza S, Klein RG, Bessler A, Malloy P, \& LaPadula M. (1998). Adult Psychiatric Status of Hyperactive Boys Grown Up. American Journal of Psychiatry, 155(4), 493-498. [PubMed: 9545994]

Meinzer MC, Pettit JW, \& Viswesvaran C. (2014). The co-occurrence of attention-deficit/hyperactivity disorder and unipolar depression in children and adolescents: A meta-analytic review. Clinical Psychology Review, 34(8), 595-607. [PubMed: 25455624]

Meinzer MC, Pettit JW, Waxmonsky JG, Gnagy E, Molina BS, \& Pelham WE (2016). Does childhood attention-deficit/hyperactivity disorder (ADHD) predict levels of depressive symptoms during emerging adulthood? Journal of Abnormal Child Psychology, 44(4), 787-797. [PubMed: 26272531]

Merrill BM, Molina BS, Coxe S, Gnagy EM, Altszuler AR, Macphee FL, Morrow AS, Trucco EM, \& Pelham WE (2019). Functional Outcomes of Young Adults with Childhood ADHD: A Latent Profile Analysis. Journal of Clinical Child \& Adolescent Psychology, 1-14.

Modesto-lowe V, \& Kranzler HR (1999). Diagnosis and treatment of alcohol-dependent patients with comorbid psychiatric disorders. Alcohol Research and Health, 144-149. [PubMed: 10890809]

Molina BSG, Pelham WE, Cheong J, Marshal MP, Gnagy EM, \& Curran PJ (2012). Childhood attention-deficit/hyperactivity disorder (ADHD) and growth in adolescent alcohol use: The roles of functional impairments, ADHD symptom persistence, and parental knowledge. Journal of Abnormal Psychology, 121(4), 922-935. [PubMed: 22845650]

Molina BS, \& Pelham WE (2003). Childhood predictors of adolescent substance use in a longitudinal study of children with ADHD. Journal of Abnormal Psychology, 112(3), 497. [PubMed: 12943028]

Molina BS, Pelham WE, Gnagy EM, Thompson AL, \& Marshal MP (2007). Attention-deficit/ hyperactivity disorder risk for heavy drinking and alcohol use disorder is age specific. Alcoholism: Clinical and Experimental Research, 31(4), 643-654.

Molina BSG, Walther CAP, Cheong J, Pedersen S, Gnagy EM, \& Pelham WE (2014). Heavy Alcohol Use in Early Adulthood as a Function of Childhood ADHD: Developmentally-Specific Mediation by Social Impairment and Delinquency. Experimental and Clinical Psychopharmacology, 22(2), 110-121. [PubMed: 24611838]

Muthén, \& Muthén. (1998). Mplus User's Guide (Eigth). Muthén \& Muthén.

Nagin DS, Jones BL, Passos VL, \& Tremblay RE (2018). Group-based multi-trajectory modeling. Statistical Methods in Medical Research, 27(7), 2015-2023. [PubMed: 29846144]

Neuman RJ, Hudziak JJ, Heath A, Reich W, Bucholz KK, Madden PA, Sun L, \& Todd RD (2001). Latent class analysis of ADHD and comorbid symptoms in a population sample of adolescent female twins. The Journal of Child Psychology and Psychiatry and Allied Disciplines, 42(7), 933942.

Pandina RJ, Johnson VL, \& White HR (2010). Peer influences on substance use during adolescence and emerging adulthood In Scheier L (Ed.), Handbook of drug use etiology: Theory, methods, and empirical findings (p. 383-401). American Psychological Association.

Patrick ME, \& Schulenberg JE (2011). How trajectories of reasons for alcohol use relate to trajectories of binge drinking: National panel data spanning late adolescence to early adulthood. Developmental Psychology, 47(2), 311. [PubMed: 21219061]

Pedersen SL, Walther CA, Harty SC, Gnagy EM, Pelham WE, \& Molina BS (2016). The indirect effects of childhood attention deficit hyperactivity disorder on alcohol problems in adulthood through unique facets of impulsivity. Addiction, 111(9), 1582-1589. [PubMed: 26999438] 
Pelham WE, Gnagy EM, Greenslade KE, \& Milich R. (1992). Teacher ratings of DSM-III-R symptoms for the disruptive behavior disorders. Journal of the American Academy of Child \& Adolescent Psychiatry, 31(2), 210-218. [PubMed: 1564021]

Pelham WE, Gnagy B, Greiner A, Hoza B, Sams S, Martin L, \& Wilson T. (1996). A summer treatment program for children with ADHD. Model Programs for Service Delivery for Child and Family Mental Health, 193-213.

Radloff LS (1977). The CES-D scale: A self-report depression scale for research in the general population. Applied Psychological Measurement, 1(3), 385-401.

Roy A, Hechtman L, Arnold LE, Sibley MH, Molina BS, Swanson JM, Howard AL, Vitiello B, Severe JB, \& Jensen PS (2016). Childhood factors affecting persistence and desistence of attentiondeficit/hyperactivity disorder symptoms in adulthood: Results from the MTA. Journal of the American Academy of Child \& Adolescent Psychiatry, 55(11), 937-944. [PubMed: 27806861]

Semeijn EJ, Comijs HC, Kooij JJS, Michielsen M, Beekman ATF, \& Deeg DJH (2015). The role of adverse life events on depression in older adults with ADHD. Journal of Affective Disorders, 174, 574-579. [PubMed: 25562670]

Smith GT, Guller L, \& Zapolski T. (2013). A comparison of two models of urgency: Urgency predicts both rash action and depression in youth. Clinical Psychological Science, 1(3), 266-275. [PubMed: 25419495]

Snyder HR (2013). Major Depressive Disorder is Associated with Broad Impairments on Neuropsychological Measures of Executive Function: A Meta-Analysis and Review. Psychological Bulletin, 139(1), 81-132. [PubMed: 22642228]

Tarter RE, Kirisci L, Mezzich A, Cornelius JR, Pajer K, Vanyukov M, Gardner W, Blackson T, \& Clark D. (2003). Neurobehavioral Disinhibition in Childhood Predicts Early Age at Onset of Substance Use Disorder. American Journal of Psychiatry, 160(6), 1078-1085. [PubMed: 12777265]

Wang FL, Pedersen SL, Joseph H, Gnagy EM, Curran P, Pelham WE, \& Molina BS (2018). Role of ADHD in the Co-occurrence between Heavy Alcohol Use and Depression Trajectories in Adulthood. Alcoholism: Clinical and Experimental Research, 43(2), 342-352.

Weafer J, Milich R, \& Fillmore MT (2011). Behavioral components of impulsivity predict alcohol consumption in adults with ADHD and healthy controls. Drug and Alcohol Dependence, 113(2), 139-146. [PubMed: 20863628]

Weiss G, Hechtman L, Milroy T, \& Perlman T. (1985). Psychiatric status of hyperactives as adults: a controlled prospective 15-year follow-up of 63 hyperactive children. Journal of the American Academy of Child Psychiatry, 24(2), 211-220. [PubMed: 3989165]

Zeileis A, Kleiber C, \& Jackman S. (2008). Regression Models for Count Data in R. Journal of Statistical Software 27(8). URL http://www.jstatsoft.org/v27/i08/ 


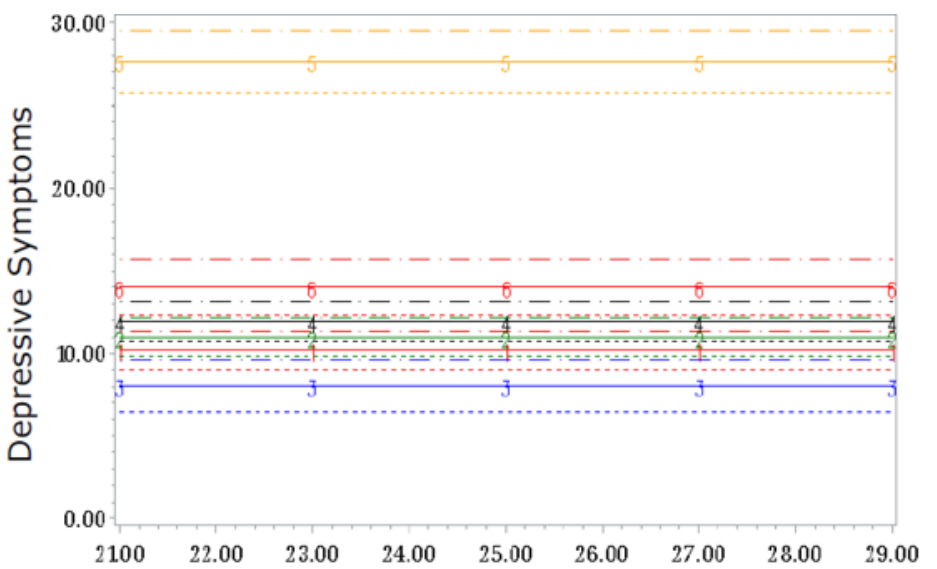

Group Percents

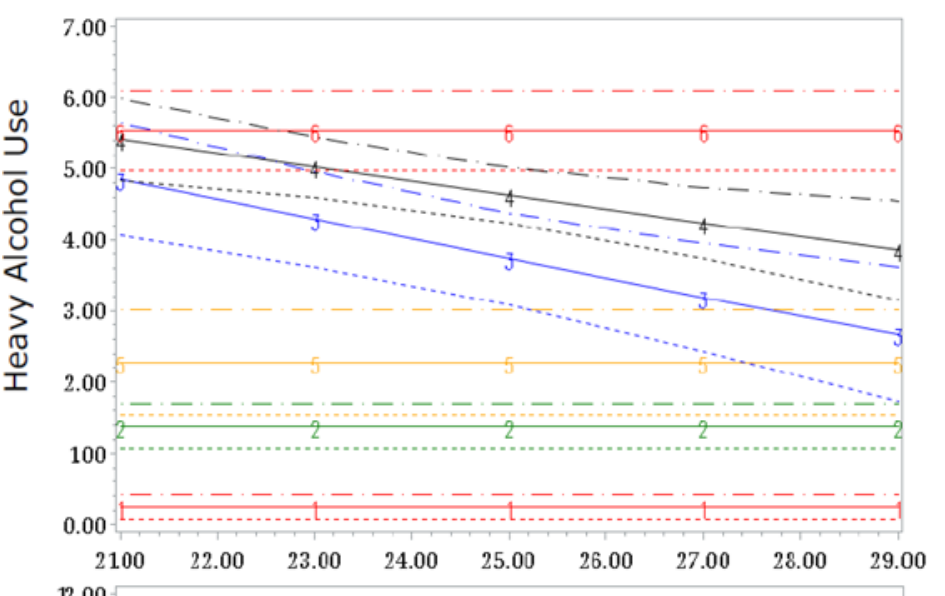

$1+1 \pm 20.9$

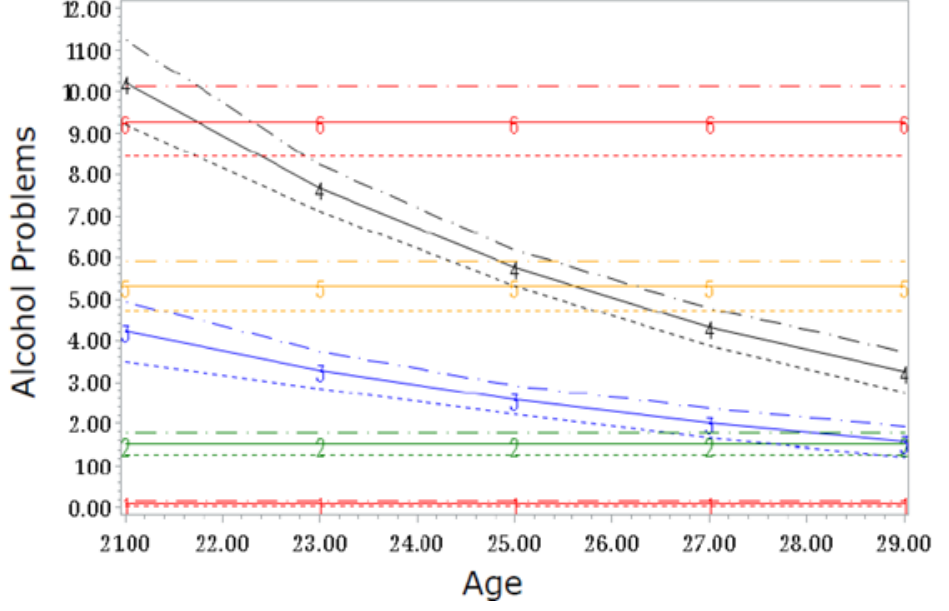

$2_{2-2}^{2-6} 24$

3 3-3 18.1

$4+215$

$-$

$55^{5-5} 5.6$

$5-\cdots$

$6+9.3$

6

Figure 1.

Trajectory plots for six-group model. $N=320$. Upper and lower dashed lines: $95 \%$ confidence intervals. Groups: (1) virtually no problems; (2) low problems; (3) moderatedecreasing drinkers with lowest depression; (4) severe-decreasing drinkers with low depression; (5) comorbid primarily depression; (6) comorbid primarily alcohol. 


\section{Table 1}

\section{Zero-order Correlations}

\begin{tabular}{|c|c|c|c|c|c|c|}
\hline & Childhood ADHD & $\begin{array}{l}\text { Adulthood } \\
\text { Persistent ADHD }\end{array}$ & Sex & Race & $\begin{array}{l}\text { Parental } \\
\text { Education }\end{array}$ & $\begin{array}{l}\text { Childhood } \\
\text { Conduct } \\
\text { Disorder }\end{array}$ \\
\hline Childhood ADHD & 1 & & & & & \\
\hline Persistent ADHD & $0.41^{* *}$ & 1 & & & & \\
\hline Sex & 0.01 & 0.07 & 1 & & & \\
\hline Race & -0.02 & -0.11 & -0.06 & 1 & & \\
\hline Parental Education & -0.09 & -0.02 & 0.002 & 0.07 & 1 & \\
\hline Childhood Conduct Disorder & $0.42 *$ & 0.04 & $-0.14^{*}$ & 0.04 & $-0.12^{*}$ & 1 \\
\hline Heavy Drinking-age 21 & $-0.12^{*}$ & -0.02 & $-0.19^{* *}$ & $0.17^{* * *}$ & -0.02 & 0.01 \\
\hline Heavy Drinking-age 23 & $-0.12^{*}$ & 0.05 & $-0.15^{* *} / \mathrm{I}$ & $0.13^{*}$ & 0.02 & -0.07 \\
\hline Heavy Drinking-age 25 & $-0.19^{* *}$ & -0.03 & $-0.12^{*}$ & $0.19^{* *}$ & -0.05 & -0.10 \\
\hline Heavy Drinking-age 27 & $-0.15^{*}$ & 0.01 & $-0.15^{* *}$ & $0.13^{*}$ & 0.02 & $-0.12^{*}$ \\
\hline Heavy Drinking-age 29 & $-0.16^{* *}$ & 0.05 & $-0.14^{*}$ & $0.20^{* * *}$ & $0.15^{*}$ & -0.11 \\
\hline Alcohol Problems-age 21 & $-0.21^{* *}$ & -0.02 & -0.09 & 0.10 & 0.03 & $-0.21^{* *}$ \\
\hline Alcohol Problems-age 23 & $-0.13^{*}$ & 0.07 & -0.11 & $0.14^{*}$ & -0.02 & $-0.13^{*}$ \\
\hline Alcohol Problems-age 25 & $-0.17^{* *}$ & 0.09 & -0.03 & 0.08 & 0.02 & -0.08 \\
\hline Alcohol Problems-age 27 & -0.07 & 0.09 & -0.08 & $0.12 *$ & 0.03 & -0.04 \\
\hline Alcohol Problems-age 29 & -0.10 & $0.16^{* *}$ & -0.06 & $0.11^{*}$ & 0.11 & 0.03 \\
\hline Depression-age 21 & $0.21^{* *}$ & $0.30^{* * *}$ & $0.17^{* * *}$ & $-0.14^{*}$ & $-0.14^{*}$ & 0.06 \\
\hline Depression-age 23 & $0.15^{*}$ & $0.17^{* * *}$ & 0.01 & $-0.15^{* *}$ & -0.08 & 0.03 \\
\hline Depression-age 25 & 0.04 & $0.14^{*}$ & -0.01 & $-0.13^{*}$ & -0.11 & -0.07 \\
\hline Depression-age 27 & 0.02 & 0.06 & 0.001 & -0.02 & $-0.12^{*}$ & -0.09 \\
\hline Depression-age 29 & $0.21^{* *}$ & $0.22^{* *}$ & -0.003 & -0.01 & 0.02 & $0.12^{*}$ \\
\hline $\begin{array}{l}\text { Notes. } \\
* \begin{array}{l}* \\
p<0.05\end{array}\end{array}$ & & & & & & \\
\hline
\end{tabular}




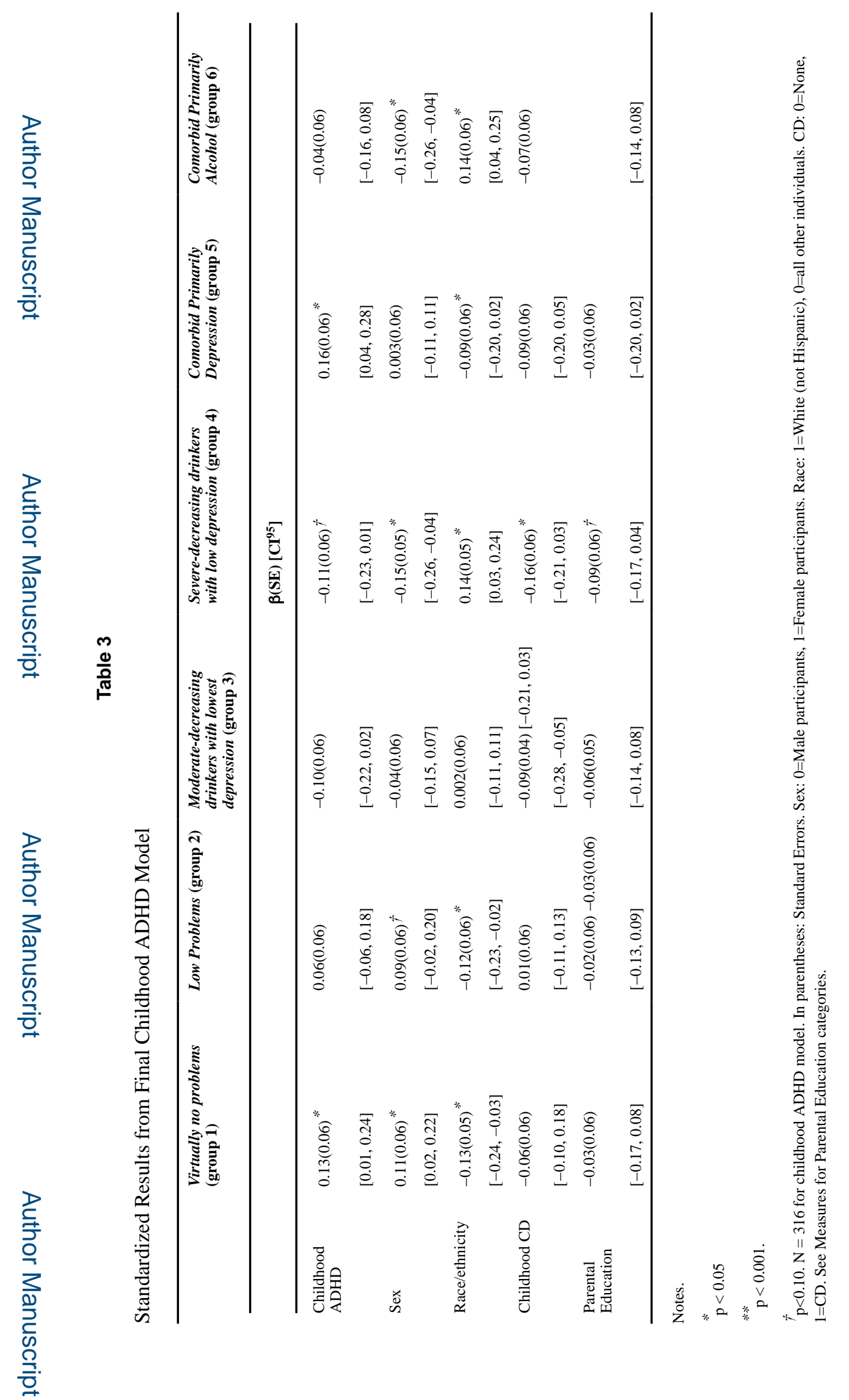

J Abnorm Child Psychol. Author manuscript; available in PMC 2021 October 01. 


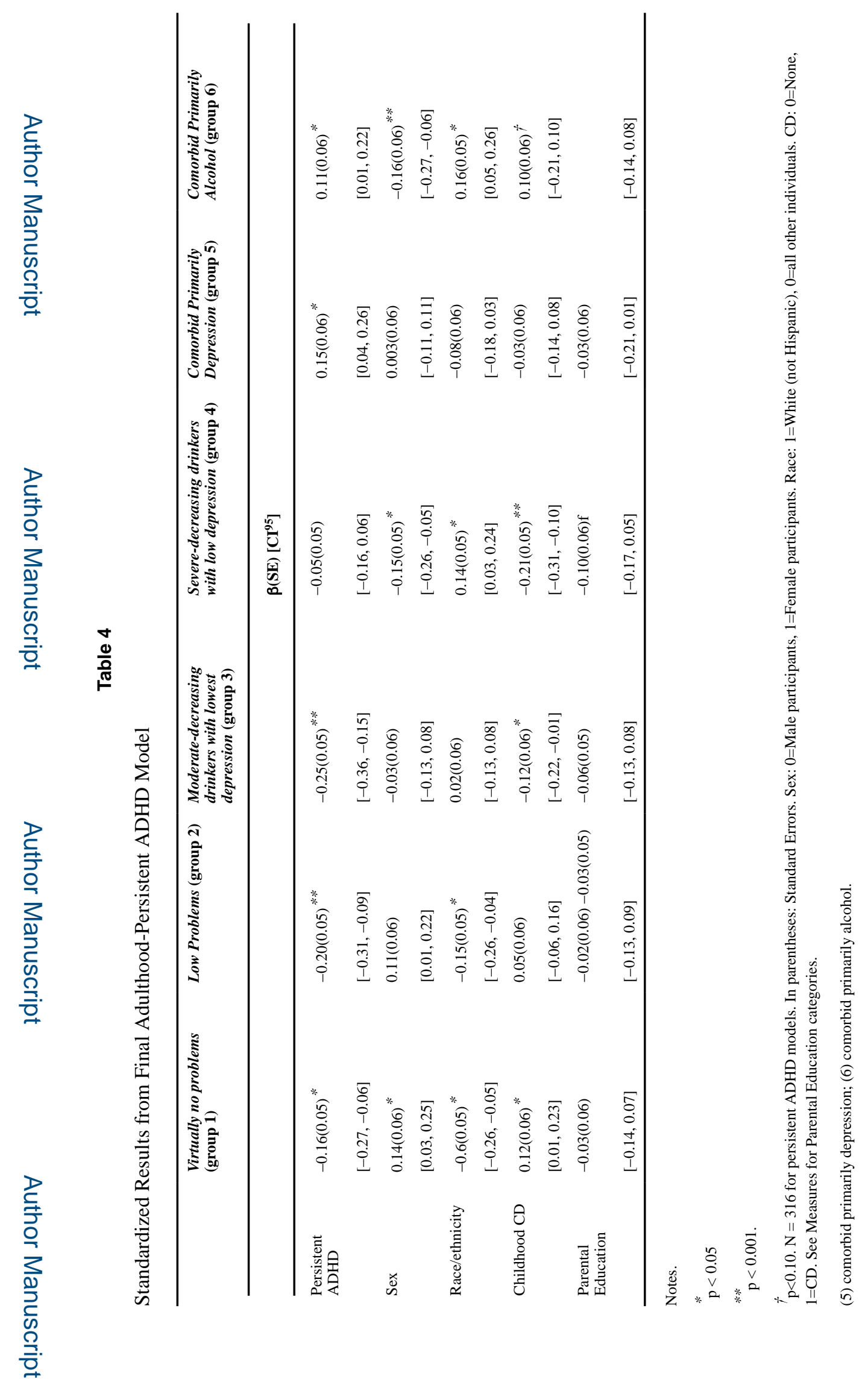

J Abnorm Child Psychol. Author manuscript; available in PMC 2021 October 01. 